OPEN ACCESS

Edited by:

Jan Polák,

Charles University, Czechia

Reviewed by:

Lin Liu,

Peking University Health Science

Centre, China

Ming Gao,

Northwest Women's and Children's

Hospital, China

*Correspondence:

Xia Li

lixia@csu.edu.cn

tThese authors have contributed equally to this work

Specialty section: This article was submitted to Clinical Diabetes,

a section of the journa

Frontiers in Public Health

Received: 20 December 2019 Accepted: 20 April 2020

Published: 29 May 2020

Citation:

Li J, Huang J, Zheng L and Li X (2020) Application of Artificial Intelligence in

Diabetes Education and Management:

Present Status and Promising

Prospect. Front. Public Health 8:173.

doi: 10.3389/fpubh.2020.00173

\section{Application of Artificial Intelligence in Diabetes Education and Management: Present Status and Promising Prospect}

\author{
Juan $\mathrm{Li}^{1,2,3+}$, Jin Huang ${ }^{1 \dagger}$, Lanbo Zheng ${ }^{4}$ and Xia $\mathrm{Li}^{2,3 *}$ \\ ${ }^{1}$ Clinical Nursing Teaching and Research Section, The Second Xiangya Hospital, Changsha, China, ${ }^{2}$ Department of \\ Metabolism and Endocrinology, The Second Xiangya Hospital, Central South University, Changsha, China, ${ }^{3}$ National Clinical \\ Research Center for Metabolic Diseases, Changsha, China, ${ }^{4}$ School of Logistics Engineering, Wuhan University of \\ Technology, Wuhan, China
}

Despite the rapid development of science and technology in healthcare, diabetes remains an incurable lifelong illness. Diabetes education aiming to improve the self-management skills is an essential way to help patients enhance their metabolic control and quality of life. Artificial intelligence (Al) technologies have made significant progress in transforming available genetic data and clinical information into valuable knowledge. The application of Al tech in disease education would be extremely beneficial considering their advantages in promoting individualization and full-course education intervention according to the unique pictures of different individuals. This paper reviews and discusses the most recent applications of $\mathrm{Al}$ techniques to various aspects of diabetes education. With the information and evidence collected, this review attempts to provide insight and guidance for the development of prospective, data-driven decision support platforms for diabetes management, with a focus on individualized patient management and lifelong educational interventions.

Keywords: artificial intelligence, diabetes, diabetes education, diabetes management, Al applications

\section{INTRODUCTION}

Diabetes mellitus (DM) is a long-term chronic disease. According to recently published data from the International Diabetes Federation, the number of people worldwide suffering from diabetes has reached 451 million (1). After undergoing a long period of fast economic growth and corresponding lifestyle changes, China is now facing many issues, such as demographic aging, urbanization, and the largest number of diabetes patients ( $\sim 114.4$ million) in the world (2). Characterized by an insidious onset, numerous complications, and high rates of death and disability, diabetes is an immense burden on individuals and society. Therefore, the prevention and management of diabetes is extremely important and diabetes education is an essential component. Over the past several years, increasing AI-based tools have been developed for diabetes healthcare (3). Patients are supported with more flexible and scholarly access to skills and knowledge for various aspects of diabetes self-management, including diabetes prevention, lifestyle and dietary guidance, exercise, insulin injection, and complications monitoring, to list a few. This paper reviews these advances. 


\section{THE CONCEPT OF AI AND ITS ADVANTAGES}

\section{The Concept of Al}

Artificial intelligence is an interdisciplinary concept based on the foundations of computer science, control theory, information theory, neuropsychology, philosophy, and linguistics (4). In general, AI employs artificial methods to impart intelligence on computers. The term "artificial intelligence" was proposed as early as 1956. In the studies that followed, researchers expanded the concept and proposed nutritious theories and technologies to enable human intelligence by computers. It is believed that by mimicking and improving on the functions of the human brain, AI can free humans from tedious mental and physical work.

Artificial intelligence, sometimes called machine intelligence, is a broad topic. Depending on the objectives of the diabetesrelated application, AI methodologies can be classified into three categories: exploring and discovering information, learning to use information, and extracting conclusions from information (3). Searching and designing algorithms to find potential information from databases is commonly known as "knowledge discovery in databases" (KDD). The main goal of KDD, which requires both a broad and a deep knowledge of the area of study of interest, is to identify useful and understandable information. The most representative technologies used in this type of research are the K-means, K-nearest neighbors (KNN) algorithms and hierarchical clustering. As for the learning from knowledge category, the idea is to enable a machine to learn automatically without human intervention or assistance, so that it is able to make predictions on the future status of complex systems for better decision making. This process includes all methods that involve inductive components and they are adaptively used in different cases with their own strengths. The most commonly used techniques are artificial neural networks (ANNs), support vector machines (SVMs), random forest (RF) algorithms, evolutionary algorithms (EAs), deep learning (DL), naive Bayes (NB), decision trees (DTs), and regression algorithms (RAs). The final category, extracting conclusions from information, is often conducted through expert systems. Those systems generally contain three main components: knowledge acquisition interfaces, knowledge bases of rules and information, and inference engines. Those systems process ambiguous concepts and uncertainty via information learned from previous cases and expert knowledge and provide decision-making support for new cases. The most commonly used techniques in this field include rule-based reasoning (RBR), case-based reasoning (CBR), and fuzzy logic (FL).

\section{Al Advantages in Medical Science}

The application of AI in medical science is becoming comprehensive and has yielded immense benefits. The key advantages include broad coverage, low cost, high efficiency, portability, diversity, and service-end "productivity." In addition,

Abbreviations: AI, artificial intelligence; ANN, artificial neural network; CBR, case-based reasoning; ES, expert systems; FL, fuzzy logic; GA, genetic algorithms; ML,machine learning; RBR, rule-based reasoning; SVM, support vector machines.
AI enhances the path toward full-course, individualized, and intelligent chronic disease management. Some of the most representative and active results are from medical expert systems. Through the use of AI technologies, the expert systems can make inferences and judgments based on large bodies of professional knowledge and experience that have been accumulated by medical experts, thereby emulating the decisionmaking processes used by human experts (5).

AI technologies, such as machine learning and data mining, have been increasingly integrated with medical science and are gradually becoming important drivers of medical development. AI presents unique advantages in disease diagnosis and prediction, surgical robotics, image recognition, virtual medical assistance, pharmaceutical research and development, and health management (6-11). In the field of diabetes education, AI technologies have been extensively utilized in diabetes prediction, dietary and exercise guidance, insulin injection guidance, monitoring of complications, and self-management.

\section{CURRENT STATE OF DIABETES EDUCATION/MANAGEMENT AND EXISTING PROBLEMS}

It is well-accepted that self-management is essential to reduce the risks of chronic complications in diabetes patients. Diabetes education is a system-wide and individualized way to ensure the effectiveness of patient self-management (12). Considering the variety of individual needs, goals, and life experiences, patients should be educated with fine-tuned knowledge and skills that fit their own situation. This kind of patient-oriented education has been proven to improve clinical outcomes and reduce management costs (13). The treatment guidelines used in the United States, Britain, and China all specify that a team of professionals with different areas of specialization should be established to provide education on diabetes management to patients and their family members, thereby ensuring that patients possess effective self-management skills (14-16).

The importance of diabetes self-management education was recognized in the early 1970 s to 1980 s (17). However, despite this recognition, most of the evidence relevant to this topic was obtained from before-and-after comparisons of patients with uncontrolled diabetes or from non-randomized control studies $(18,19)$. Diabetes education helps patients to develop self-management skills to manage their own incorrect behaviors, and therefore, various methods are used to customize patientoriented programs. While patients are the greatest beneficiaries of their educational intervention, a recent study that covered 12 quantitative and qualitative methods revealed that a relatively large percentage of patients refuse to participate in educational programs. The reasons include lack of time, excessive class time, class contents that are not suitable for the patient, and unaffordable travel expenses (20). In addition, one offline diabetes education program can only serve $10-20$ patients at a time, while there is a worldwide shortage of diabetes specialists, nurses, and diabetes education personnel (21). This suggests that new methods and models of educational intervention should 
be explored to improve efficiency and coverage such that the long-term and patient-specific requirements can be satisfied.

In recent years, diversified models for diabetes education programs have been designed and conducted, including diabetes management applications (apps), summer camp activities, interventions by interdisciplinary teams, remote interventions, sequential health education, peer education, large-class education, and small group education. Although AI platforms based on apps and web services are becoming an effective alternative that is able to provide education to a massive number of diabetes patients, the percentage of physicians and patients willing to use this technology in diabetes management remains relatively low (22). One of the major flaws is that the functions do not satisfy patients' needs. The underlying reason is that the apps were designed without the participation of medical professionals. Without incorporating recommendations from physicians on diagnosis or treatment, the apps lack the high-quality proof of effectiveness that can be obtained from clinical trials (23).

The task of designing and implementing diabetes educational systems is very complicated and requires that interdisciplinary experts work together to obtain the best outcome. Although various educational methods with more or less pros and cons exist, problems such as how to use existing technologies to optimize educated decisions, how to provide patient-specific guidance and individualized management plans, and how to conduct long-term, effective educational intervention for lifelong chronic diseases are the focus of what needs to be addressed by diabetes education.

\section{CURRENT STATUS OF AI APPLICATIONS IN DIABETES EDUCATION AND MANAGEMENT}

Compared with the conventional education methods, AIbased education offers significant advantages including low costs, easy implementation, broad coverage, flexible doctorpatient interaction, avoidance of repeated efforts, reduction in the workload of medical personnel, and an enhancement in effectiveness. Current AI technologies applied to diabetes education mainly focus on diabetes prediction, lifestyle guidance, insulin injection guidance, blood sugar monitoring, selfmanagement, and complication monitoring. Here, we try to illustrate as comprehensive as possible the existing AI methods that have been applied in diabetes management from various aspects.

\section{Diabetes Prediction}

To predict the risk of adults developing type 2 diabetes, British scholars performed a systematic assessment of 94 existing type 2 diabetes risk assessment models and scoring standards using both quantitative and qualitative methods. They also selected some of these models and scoring methods and applied them in practice. The experimental results proved the stability of a considerable proportion of the risk assessment approaches and verified these approaches in different patient groups (24). A study by Mani et al.
(25) used various machine learning algorithms in conjunction with electronic medical record data from 2,000 patients with type 2 diabetes to predict the risk of diabetes. It was found that the area under the curve (AUC) values of 6-months and 1-year predictions were $<0.8$. This indicated the feasibility of using electronic medical record data to automatically predict diabetes and identify persons at a high risk of diabetes. Fu et al. developed a risk assessment model for postprandial hyperglycemia that can be used to identify persons at a high risk for this condition to ultimately reduce the number of people who must take an oral glucose tolerance test (OGTT) (26). In this sense, we hold this thought that different assessment models should be developed for different races and AI models focusing on postprandial hyperglycemia are extremely promising to improve the efficiency of diabetes prediction.

\section{Lifestyle Guidance for Diabetes Patients}

Understanding the carbohydrate content of individual diets and how carbohydrates affect blood glucose is one of the cornerstones of diabetes treatment. To appropriately adjust an insulin dose, the total amount of carbohydrates ingested by the patient must be calculated. As early as the 1990s, some scholars used a computerized device (a food meter) to help 21 diabetes patients record the types and amounts of food and beverages they consumed during a 1-week period (27). This approach shed light on investigating dietary types and characteristics of patients so as to provide individualized insulin dose selection advice for better blood glucose management. An experimental study indicated that compared with relying solely on carbohydrate levels to perform insulin dose calculation, taking fat and protein into consideration may lead to a significant reduction in hemoglobin A1C (HbAlc) levels (28). Software applications that use graphic analysis technologies to quickly and directly analyze dietary content are under development. The proposed software allows patients to quickly obtain relevant nutrition information, including carbohydrate content and calories, by taking smartphone photos and will thereby help in their nutritional choices (29). Recently, a study by Oka et al. (30), employed a deep learning and remote communications technology to analyze photographs of patients' meals and thereby automatically assess nutrient intake. The results of a 12-months randomized controlled experiment showed that the average variation in HbAlc level was $0.3 \%$ with a standard deviation of $0.3 \%$. This indicated that nutritional intervention through automated AI support yields results similar to those obtained with face-to-face nutritional intervention support from a human dietitian. These methods can therefore greatly reduce the load on the workforce of human experts, while simultaneously enhancing the effectiveness of dietary guidance for diabetics.

Zeevi et al. (31) designed a machine learning algorithm that was able to integrate the blood parameters, dietary habits, blood measurements, and physical activities of an 800-person cohort and verified that the algorithm was able to accurately predict postprandial glycemic responses to real-life meals for the individuals in a 100-person cohort. The results of this experiment indicated that individualized diets can successfully reduce postprandial glucose elevation and its metabolic consequences. 
Proper exercise plays an important role in blood sugar control and reduce the risk of cardiovascular events. The Action for Health in Diabetes (Look AHEAD) trial relied on machine learning to reanalyze data and confirmed that type 2 diabetes patients can benefit from intensive lifestyle intervention involving weight loss, which can also reduce the incidence of cardiovascular events (32). During the early stage of diabetes, commitment to a healthy diet and weight loss can be employed as preventive measures. Everett et al. (33) proposed a decision support system using machine learning and found that this system was able to promote compliance with physical activity and weight loss goals of the users and therefore reduce their risk of developing diabetes. Jacobs et al. (34) proposed a regression model that could be used to automatically analyze the exercise levels of patients wearing accelerometers and heart monitors and monitor changes in glucose levels that occurred while the subjects were exercising. The results from an assessment of 13 type 1 diabetes patients had a sensitivity of $97.2 \%$ and a specificity of $99.5 \%$.

With the technical progress of picture visualization and the widespread use of wearable devices, we believe that AI would play a crucial role in personalized lifestyle guidance and thus help the management of diabetes.

\section{Insulin Injection Guidance}

For patients with type 1 diabetes, lifelong dependence of insulin is the only effective treatment (35). Published data from the American Diabetes Control and Complication Trial (DCCT) indicated that a $1.9 \%$ decrease in HbAlc through early intensive insulin treatment could significantly decrease the risk of complications and death $(36,37)$. Furthermore, the most recent evidence indicated that large doses of insulin may be associated with adverse cardiovascular outcomes in patients (38). Insulin is therefore a double-edged sword, and should be used in a safe and effective manner.

In theory, insulin dosage can be calculated using formulas with the following inputs: carbohydrate coefficient, insulin sensitivity coefficient, and data collected from dynamic blood sugar monitoring; however, AI technologies have been intensively applied to this aspect of diabetes management to provide better insulin usage support for patients. In a recent multicenter, randomized controlled study, Bergenstal et al. (39) used the d-Nav insulin guidance system to monitor and predict glucose levels so as to automatically advise patients appropriately on their next insulin dose. The data used by the researchers included glucose readings (sensor readings or fingertip blood glucose measurements), insulin dose records, dietary carbohydrate intake, and other relevant information. The algorithmic core was an event-driven machine learning method. The results indicated that $\mathrm{HbAlc}$ decreased by an average of $1.0 \%$ from baseline levels to 6 months in the intervention group and the difference compared to the control group was significant. These experimental results indicated a potential use of the d-Nav insulin guidance system for stable blood sugar control and the optimization of insulin management in type 2 diabetes patients.

In another study, Nimri et al. (40) worked on a closedloop remote monitoring system called MD-Logic. The analysis is based on fuzzy logic and a self-adaptive learning algorithm. A randomized controlled experiment involving 75 diabetes patients was performed to observe the occurrence of nocturnal hypoglycemia. This study found that the system could significantly reduce the median duration of nocturnal hypoglycemia $(P=0.004)$. Considering its effectiveness at reducing the risk of nocturnal hypoglycemia, the authors concluded that the MD-Logic system can be used safely at night by children, adolescents, and type 1 diabetes patients.

Logic-based AI tools are also utilized to provide insulin guidance. Researchers have tried to determine the effects of eating time, drinking time, and exercise time on individual glucose metabolism via case-based reasoning (CBR). This method was used to calculate an individualized insulin bolus using an insulin intravenous bolus calculator, thereby achieving optimal glucose levels in patients and optimizing insulin treatment (41).

Most recently, it was reported that an app named CamAPS FX has become the world's first available artificial pancreas app to people with type 1 diabetes in UK (42). This is a huge step for the AI application in the automomic calculation of insulin dosage and would tremendously improve the metabolic control of insulin-treated patients with diabetes.

\section{Blood Sugar Monitoring}

Numerous studies have verified that continuous glucose monitoring (CGM) employing an intelligent algorithm combined with insulin pump therapy can help patients achieve a better understanding of their blood sugar fluctuations. Compared with traditional monitoring methods, CGMs provide the whole picture of blood sugar and can better detect occult hypoglycemia, making it an excellent means of observing blood sugar fluctuations and enhancing self-management ability $(43,44)$. A recent study used a neural network system to perform a comparative assessment of four blood sugar prediction models used by type 1 diabetes patients. The researchers used data from 10 type 1 diabetes patients, including data on physical activity obtained by means of sensors, and found that the model was able to effectively predict the risk of hypoglycemia (45). In the United States, the WellDoc diabetes management system, a smartphone software application, has been approved by the FDA and is covered by most types of medical insurance. Doctors can use this system in conjunction with patients' blood sugar diaries to provide individualized feedback and recommendations via real-time online communication. Doctors can also prescribe the app to patients so that they can continue to record their blood sugar levels in real time and upload dietary and drug treatment information. Quinn et al. (46) used this app to perform a randomized control trial involving type 2 diabetes patients and found that patients using the smartphone app to manage their diabetes had A1c levels that were 1.2\% lower than those of the control group who received drug treatment alone. In China, Ling et al. (47) assessed the effectiveness of the mobile health app TangTangQuan (TTQ) in the management of type 1 diabetes; patients used the TTQ system to record and upload their diabetes diaries. The patients and their companions or family members were able to provide their comments, share their own experiences, and interact with researchers. The results 
TABLE 1 | Examples of the most representative publications on Al applied to diabetes education.

\begin{tabular}{|c|c|c|c|}
\hline Method & Application & Description & References \\
\hline $\begin{array}{l}\text { SVM } \\
\text { ML }\end{array}$ & Diabetes prediction & $\begin{array}{l}\text { SVM are recently one of the most popular and flexible ML algorithms used for classification, they are } \\
\text { being used to discover valuable knowledge from large databases such as to test the feasibility of using } \\
\text { data collected in electronic medical records for development of effective models for diabetes risk } \\
\text { forecasting. But the approach is tolerant to a reasonable number of false positives. }\end{array}$ & $(24,25)$ \\
\hline $\begin{array}{l}\text { ANN } \\
\text { DL }\end{array}$ & $\begin{array}{l}\text { Diet Guidance } \\
\text { Retinopathy assessment } \\
\text { Exercise guidance }\end{array}$ & $\begin{array}{l}\text { The most widely used techniques are artificial neural networks (ANNs). ANN are based on interconnected } \\
\text { neurons, that means, the human brain function. A deep learning algorithm (DL), can be considered and } \\
\text { evolution of ANN. For example, based on the techniques of ANN, then proposed a regression model that } \\
\text { could be used to automatically analyze the exercise levels of patients wearing accelerometers and heart } \\
\text { monitors and monitor changes in glucose levels that occurred while the subjects were exercising. }\end{array}$ & $\begin{array}{c}(28,30,32,33) \\
(50,51) \\
(34)\end{array}$ \\
\hline CBR & $\begin{array}{l}\text { Insulin dose } \\
\text { recommendation }\end{array}$ & $\begin{array}{l}\text { Case-based reasoning (CBR) is used to calculate an individualized insulin bolus using an insulin } \\
\text { intravenous bolus calculator, thereby achieving optimal glucose levels in patients and optimizing insulin } \\
\text { treatment. CBR learns from experiences of past similar meals, which are described in cases through a } \\
\text { set of parameters (e.g., time of meal, exercise). However, there are some limitations of CBR, as its } \\
\text { application needs to get a large sample size and is often excessively time-consuming. }\end{array}$ & $(39,41)$ \\
\hline DT & Diabetes management & $\begin{array}{l}\text { DT is most often created based on a learning algorithm. By recording information on diet, exercise, } \\
\text { pharmaceutical use, and blood sugar levels, the application of } \mathrm{DL} \text { in the systems can combine patient- } \\
\text { and physician-support tools for the purpose of improving disease outcomes. }\end{array}$ & $(46,48,49)$ \\
\hline
\end{tabular}

of this study indicated that TTQ can be used as a means of intervention to promote active communication with type 1 diabetes patients and family members and can help support diabetes self-management education.

Thanks to the rapid development of the Internet and AI technologies, decision support systems (DSSs) are already being used in the management of diabetes treatment. These systems can record information on diet, exercise, pharmaceutical use, and blood sugar levels and can be combined with patient- and physician-support tools for the purpose of improving disease outcomes $(48,49)$. The use of AI technology and online applications in diabetes management is becoming increasingly effective in both the United States and China $(48,49)$.

\section{Monitoring of Diabetes Complications}

The most common complications of diabetes include vascular pathologies and peripheral neuropathies. Scholars who assessed adult retinal fundus photographs found that detection of diabetic retinopathies by a deep learning algorithm achieved sensitivity and specificity of more than $93 \%$ (50). In a retrospective analysis of 9,939 posterior pole photographs from 2,740 diabetes patients, Takahashi et al. (51) confirmed that deep learning can be used to assess diabetic retinopathies. Based on their results, Takahashi et al. presented a new AI disease grading system that can be used to grade the severity of diabetic retinopathies.

Likewise, a mobile app called "FootSnap" was developed to standardize diabetic foot images (52). Through assessments of various scenarios presented by different practitioners, a trial involving 60 patients was conducted to test the stability of FootSnap. The Jaccard similarity index (JSI) was used to confirm the reproducibility of the foot images. The resulting JSI values for diabetic feet were $0.89-0.91$, with values of $0.93-0.94$ for the control group, which suggested that the app had excellent reliability. Kaabouch et al. (53) used asymmetry analysis in conjunction with a genetic algorithm to analyze thermal images and thereby facilitate the early detection of foot ulcerations and the assessment of skin integrity. This study found that the tested technology was a feasible means of detecting inflammation and could effectively predict potential foot ulcerations.

Katigari et al. (54) developed an expert system based on fuzzy logic and applied it to the medical records of 244 patients diagnosed with diabetic neuropathies; they found that the system could intelligently determine the severity of diabetic neuropathies with a sensitivity of $89 \%$, a specificity of $98 \%$, and an accuracy of $93 \%$.

With the development of information systems and technologies, the digital data collected from diabetes patients is growing exponentially. From the above observations, it is clear that AI technologies employing complex and precise methods can serve as useful management tools in processing diabetes databases to derive valuable information. Accordingly, AI plays a key role in these relevant systems as an auxiliary routine management tool for diabetes patients.

\section{CONCLUSIONS}

We have summarized that the most representative applications of artificial intelligence in diabetes education and management (Table 1). As described above, the diabetes education and management is an essential means of improving the quality 
of disease management. As a consequence, the integration of education and management approaches with mobile health and AI technologies has become an unstoppable trend. However, we've noticed that there are cons and pros of the wide range of AI-based methods. However, the applicability of one algorithm is problem and data specific. For example, in diabetes classification analysis, based on the classification criteria and characteristic distribution of data, some instances may generate good results with directly applying standard methods supplied by common data analysis tools. However, there are also situations where more advanced models are to be developed to infer a clearer layout of the analyzing object. We will proceed with discussing and comparing the strengths and drawbacks of some well-known AIbased methods when certain standards and benchmark data sets are prepared in our future work.

In conclusion, the use of AI in educational interventions, while promising, faces certain challenges. In spite of the fact that research on the use of digital and intelligent tools in diabetes management is expanding rapidly, most relevant studies lack sufficient numbers of samples or fail to determine whether the results of the tested intervention have clinical significance. To fully and effectively realize AI-based models and establish individualized educational intervention strategies for patients with different needs, it is necessary to achieve the following: (1) gather large amounts of patient data and establish individualized patient data profiles, (2) establish in-depth crossover between the professional knowledge of physicians and AI technologies, (3) continuously update and enlarge the existing knowledge bases,

\section{REFERENCES}

1. Cho NH, Shaw JE, Karuranga S, Huang Y, Da Rocha Fernandes JD, Ohlrogge AW, et al. IDF diabetes atlas: global estimates of diabetes prevalence for 2017 and projections for 2045. Diabetes Res Clin Pract. (2018) 138:271-81. doi: 10.1016/j.diabres.2018.02.023

2. Xu Y, Wang L, He J, Bi Y, Li M, Wang T, et al. Prevalence and control of diabetes in Chinese adults. JAMA. (2013) 310:948-59. doi: 10.1001/jama.2013.168118

3. Contreras I, Vehi J. Artificial intelligence for diabetes management and decision support: literature review. J Med Internet Res. (2018) 20:e10775. doi: $10.2196 / 10775$

4. Russell SJ, Norvig P. Artificial Intelligence: A Modern Approach. Malaysia: Pearson Education Limited (2016).

5. Sadikov A, Groznik V, Mozina M, Zabkar J, Nyholm D, Memedi M, et al. Feasibility of spirography features for objective assessment of motor function in Parkinson's disease. Artif Intelligence Med. (2017) 81:54-62. doi: 10.1016/j.artmed.2017.03.011

6. Das N, Topalovic M, Janssens W. Artificial intelligence in diagnosis of obstructive lung disease: current status and future potential. Curr Opin Pulm Med. (2018) 24:117-23. doi: 10.1097/MCP.000000000 0000459

7. Camarillo DB, Krummel TM, Salisbury JK, Jr. Robotic technology in surgery: past, present, and future. Am J Surg. (2004) 188(4A Suppl.):2s-15s. doi: 10.1016/j.amjsurg.2004.08.025

8. Gargeya R, Leng T. Automated identification of diabetic retinopathy using deep learning. Ophthalmology. (2017) 124:962-9. doi: 10.1016/j.ophtha.2017.02.008

9. Talbot TB, Sagae K, John B, Rizzo AA. Sorting out the virtual patient: how to exploit artificial intelligence, game technology and sound educational practices to create engaging role-playing simulations. Int
(4) perform standardized, randomized control studies involving clinical practice, and (5) involve both clinicians and patients jointly in a system designed to optimize effectiveness. The resolution of these challenges will depend on scientific research, regulation, and standardization of the targeted areas. In addition, other current challenges include technological, philosophical, and ethical dilemmas, as well as issues surrounding user data security and privacy, and even legal hurdles. Among these issues, information security is a new challenge for the AI era. Further efforts will be needed to promote the rapid and effective application of AI to medical fields.

Despite these challenges, the medical applications of AI have been developing at an extremely rapid rate, and the prospects and research value of these applications cannot be denied. The future use of integrated and comprehensive applications of individual AI technologies in diabetes education can provide full-course, individualized, and intelligent education and thereby provide patients with a lifetime of guidance and protection.

\section{AUTHOR CONTRIBUTIONS}

JL wrote the manuscript. JH contributed to the definition section. $\mathrm{XL}$ provided ideas. LZ polished the article.

\section{FUNDING}

This work was supported by the National Key R\&D Program of China (2017YFC1309604).
J Gaming Comp Med Simul. (2012) 4:1-19. doi: 10.4018/jgcms.20120 70101

10. Duch W, Swaminathan K, Meller J. Artificial intelligence approaches for rational drug design and discovery. Curr Pharm Design. (2007) 13:1497-508. doi: $10.2174 / 138161207780765954$

11. Furmankiewicz M, Sołtysik-Piorunkiewicz A, Ziuzianski P. Artificial intelligence systems for knowledge management in e-health: the study of intelligent software agents. In: Latest Trends on Systems: The Proceedings of 18th International Conference on Systems. Santorini Island, Greece (2014).

12. Funnell MM, Brown TL, Childs BP, Haas LB, Hosey GM, Jensen B, et al. National standards for diabetes self-management education. Diabetes Care. (2009) 32 Suppl 1:S87-94. doi: 10.2337/dc09-S087

13. Li R, Zhang P, Barker LE, Chowdhury FM, Zhang X. Cost-effectiveness of interventions to prevent and control diabetes mellitus: a systematic review. Diabetes Care. (2010) 33:1872-94. doi: 10.2337/dc10-0843

14. Chiang JL, Kirkman MS, Laffel LM, Peters AL. Type 1 diabetes through the life span: a position statement of the American Diabetes Association. Diabetes Care. (2014) 37:2034-54. doi: 10.2337/dc14-1140

15. Women's NCCf, Health Cs. Diabetes (type 1 and type 2) in Children and Young People: Diagnosis and Management. London: National Institute for Health and Care Excellence (NICE) (2015).

16. Weng J, Ji L, Jia W, Lu J, Zhou Z, Zou D, et al. Standards of care for type 2 diabetes in China. Diabetes Metab Res Rev. (2016) 32:442-58. doi: $10.1002 /$ dmrr.2827

17. Muhlhauser I, Jorgens V, Berger M, Graninger W, Gurtler W, Hornke L, et al. Bicentric evaluation of a teaching and treatment programme for type 1 (insulin-dependent) diabetic patients: improvement of metabolic control and other measures of diabetes care for up to 22 months. Diabetologia. (1983) 25:470-6. doi: 10.1007/BF00284453

18. Pieber TR, Brunner GA, Schnedl WJ, Schattenberg S, Kaufmann P, Krejs GJ. Evaluation of a structured outpatient group education 
program for intensive insulin therapy. Diabetes Care. (1995) 18:625-30. doi: $10.2337 /$ diacare.18.5.625

19. Hopkins D, Lawrence I, Mansell P, Thompson G, Amiel S, Campbell $\mathrm{M}$, et al. Improved biomedical and psychological outcomes 1 year after structured education in flexible insulin therapy for people with type 1 diabetes: the U.K. DAFNE experience. Diabetes Care. (2012) 35:1638-42. doi: $10.2337 / \mathrm{dc} 11-1579$

20. Horigan G, Davies M, Findlay-White F, Chaney D, Coates V. Reasons why patients referred to diabetes education programmes choose not to attend: a systematic review. Diabetic Med. (2017) 34:14-26. doi: 10.1111/dme.13120

21. Zurn P, Dal Poz MR, Stilwell B, Adams O. Imbalance in the health workforce. Hum Resour Health. (2004) 2:13. doi: 10.1186/1478-4491-2-13

22. Boulos MN, Brewer AC, Karimkhani C, Buller DB, Dellavalle RP. Mobile medical and health apps: state of the art, concerns, regulatory control and certification. Online J Public Health Inform. (2014) 5:229. doi: 10.5210/ojphi.v5i3.4814

23. Zhang Y, Li X, Luo S, Liu C, Xie Y, Guo J, et al. Use, perspectives, and attitudes regarding diabetes management mobile apps among diabetes patients and diabetologists in China: national web-based survey. JMIR mHealth uHealth. (2019) 7:e12658. doi: 10.2196/12658

24. Noble D, Mathur R, Dent T, Meads C, Greenhalgh T. Risk models and scores for type 2 diabetes: systematic review. BMJ. (2011) 343:d7163. doi: 10.1136/bmj.d7163

25. Mani S, Chen Y, Elasy T, Clayton W, Denny J. Type 2 diabetes risk forecasting from EMR data using machine learning. AMIA. (2012) 2012:606-15.

26. Fu Q, Sun M, Tang W, Wang Z, Cao M, Zhu Z, et al. A Chinese risk score model for identifying postprandial hyperglycemia without oral glucose tolerance test. Diabetes Metab Res Rev. (2014) 30:284-90. doi: 10.1002/dmrr.2490

27. Rivellese AA, Ventura MM, Vespasiani G, Bruni M, Moriconi V, Pacioni $D$, et al. Evaluation of new computerized method for recording 7day food intake in IDDM patients. Diabetes Care. (1991) 14:602-4. doi: 10.2337/diacare.14.7.602

28. Pankowska E, Blazik M, Groele L. Does the fat-protein meal increase postprandial glucose level in type 1 diabetes patients on insulin pump: the conclusion of a randomized study. Diab Technol Therap. (2012) 14:16-22. doi: 10.1089/dia.2011.0083

29. Froisland $\mathrm{DH}$, Arsand E. Integrating visual dietary documentation in mobile-phone-based self-management application for adolescents with type 1 diabetes. J Diab Sci Technol. (2015) 9:541-8. doi: 10.1177/19322968155 76956

30. Oka R, Nomura A, Yasugi A, Kometani M, Gondoh Y, Yoshimura $\mathrm{K}$, et al. study protocol for the effects of artificial intelligence (AI)supported automated nutritional intervention on glycemic control in patients with type 2 diabetes mellitus. Diab Therapy. (2019) 10:1151-61. doi: 10.1007/s13300-019-0595-5

31. Zeevi D, Korem T, Zmora N, Israeli D, Rothschild D, Weinberger A, et al. Personalized nutrition by prediction of glycemic responses. Cell. (2015) 163:1079-94. doi: 10.1016/j.cell.2015.11.001

32. Baum A, Scarpa J, Bruzelius E, Tamler R, Basu S, Faghmous J. Targeting weight loss interventions to reduce cardiovascular complications of type 2 diabetes: a machine learning-based post-hoc analysis of heterogeneous treatment effects in the Look AHEAD trial. Lancet Diab Endocrinol. (2017) 5:808-15. doi: 10.1016/S2213-8587(17)30176-6

33. Everett E, Kane B, Yoo A, Dobs A, Mathioudakis N. A novel approach for fully automated, personalized health coaching for adults with prediabetes: pilot clinical trial. J Med Internet Res. (2018) 20:e72. doi: 10.2196/jmi r.9723

34. Jacobs PG, Resalat N, El Youssef J, Reddy R, Branigan D, Preiser N, et al. Incorporating an exercise detection, grading, and hormone dosing algorithm into the artificial pancreas using accelerometry and heart rate. J Diab Sci Technol. (2015) 9:1175-84. doi: 10.1177/19322968156 09371

35. Li X, Cheng J, Zhou Z. Revisiting multiple models of progression of beta-cell loss of function in type 1 diabetes: significance for prevention and cure. Journal of diabetes. (2016) 8:460-9. doi: 10.1111/1753-0407. 12376
36. Nathan DM, Genuth S, Lachin J, Cleary P, Crofford O, Davis M, et al. The effect of intensive treatment of diabetes on the development and progression of long-term complications in insulin-dependent diabetes mellitus. $\mathrm{N} \mathrm{Engl} \mathrm{J}$ Med. (1993) 329:977-86. doi: 10.1056/NEJM199309303291401

37. White NH, Cleary PA, Dahms W, Goldstein D, Malone J, Tamborlane WV. Beneficial effects of intensive therapy of diabetes during adolescence: outcomes after the conclusion of the Diabetes Control and Complications Trial (DCCT). J Pediatr. (2001) 139:804-12. doi: 10.1067/mpd.2001.1 18887

38. Braffett BH, Dagogo-Jack S, Bebu I, Sivitz WI, Larkin M, Kolterman $\mathrm{O}$, et al. Association of insulin dose, cardiometabolic risk factors, and cardiovascular disease in type 1 diabetes during 30 years of follow-up in the DCCT/EDIC Study. Diabetes Care. (2019) 42:657-64. doi: 10.2337/dc1 8-1574

39. Bergenstal RM, Johnson M, Passi R, Bhargava A, Young N, Kruger DF, et al. Automated insulin dosing guidance to optimise insulin management in patients with type 2 diabetes: a multicentre, randomised controlled trial. Lancet. (2019) 393:1138-48. doi: 10.1016/S0140-6736(19)30368-X

40. Nimri R, Bratina N, Kordonouri O, Avbelj Stefanija M, Fath M, Biester T, et al. MD-Logic overnight type 1 diabetes control in home settings: a multicentre, multinational, single blind randomized trial. Diab Obesity Metabol. (2017) 19:553-61. doi: 10.1111/dom.12852

41. Pesl P, Herrero P, Reddy M, Oliver N, Johnston DG, Toumazou C, et al. Case-based reasoning for insulin bolus advice: evaluation of case parameters in a six-week pilot study. J Diab Sci Technol. (2017) 11:37-42. doi: $10.1177 / 1932296816629986$

42. University of Cambridge. World's First Artificial Pancreas App Licensed for People With Type 1 Diabetes in UK. Cambridge: Targeted News Service (2000).

43. Walsh J, Roberts R, Weber D, Faber-Heinemann G, Heinemann L. Insulin pump and CGM usage in the United States and Germany: results of a real-world survey With 985 subjects. J Diab Sci Technol. (2015) 9:1103-10. doi: $10.1177 / 1932296815588945$

44. Gomez AM, Alfonso-Cristancho R, Orozco JJ, Lynch PM, Prieto D, Saunders $\mathrm{R}$, et al. Clinical and economic benefits of integrated pump/CGM technology therapy in patients with type 1 diabetes in Colombia. Endocrinol Nutr. (2016) 63:466-74. doi: 10.1016/j.endoen.2016.10.009

45. Zarkogianni K, Mitsis K, Litsa E, Arredondo MT, Ficomicron G, Fioravanti A, et al. Comparative assessment of glucose prediction models for patients with type 1 diabetes mellitus applying sensors for glucose and physical activity monitoring. Med Biol Eng Comp. (2015) 53:1333-43. doi: 10.1007/s11517-015-1320-9

46. Quinn CC, Shardell MD, Terrin ML, Barr EA, Ballew SH, Gruber-Baldini AL. Cluster-randomized trial of a mobile phone personalized behavioral intervention for blood glucose control. Diabetes Care. (2011) 34:1934-42. doi: $10.2337 / \mathrm{dc} 11-0366$

47. Ling , Luo S, Yan J, Zheng X, Yang D, Zeng X, et al. The design and preliminary evaluation of a mobile health application TangTangQuan in management of type 1 diabetes in China. Am Diabetes Assoc. (2018) 67 (Supplement 1). doi: 10.2337/db18-860-P

48. Fico G, Arredondo MT, Protopappas V, Georgia E, Fotiadis D. Mining data when technology is applied to support patients and professional on the control of chronic diseases: the experience of the METABO platform for diabetes management. Methods Mol Biol. (2015) 1246:191-216. doi: 10.1007/978-1-4939-1985-7_13

49. Fico G, Fioravanti A, Arredondo MT, Gorman J, Diazzi C, Arcuri $\mathrm{G}$, et al. Integration of personalized healthcare pathways in an ICT platform for diabetes managements: a small-scale exploratory Study. IEEE J Biomed Health Inform. (2016) 20:29-38. doi: 10.1109/JBHI.2014.2 367863

50. Gulshan V, Peng L, Coram M, Stumpe MC, Wu D, Narayanaswamy A, et al. Development and validation of a deep learning algorithm for detection of diabetic retinopathy in retinal fundus photographs. Jama. (2016) 316:2402-10. doi: 10.1001/jama.2016.17216

51. Takahashi H, Tampo H, Arai Y, Inoue Y, Kawashima H. Applying artificial intelligence to disease staging: deep learning for improved staging of diabetic retinopathy. PLoS ONE. (2017) 12:e0179790. doi: 10.1371/journal.pone.0179790 
52. Yap MH, Chatwin KE, Ng CC, Abbott CA, Bowling FL, Rajbhandari S, et al. A new mobile application for standardizing diabetic foot images. J Diabetes Sci Technol. (2018) 12:169-73. doi: 10.1177/1932296817713761

53. Kaabouch N, Hu W-C, Chen Y, Anderson JW, Ames F, Paulson R. Predicting neuropathic ulceration: analysis of static temperature distributions in thermal images. J Biomed Optics. (2010) 15:061715. doi: 10.1117/1.35 24233

54. Katigari MR, Ayatollahi H, Malek M, Haghighi MK. Fuzzy expert system for diagnosing diabetic neuropathy. World J Diab. (2017) 8:80. doi: $10.4239 /$ wjd.v8.i2.80
Conflict of Interest: The authors declare that the research was conducted in the absence of any commercial or financial relationships that could be construed as a potential conflict of interest.

Copyright $\odot 2020$ Li, Huang, Zheng and Li. This is an open-access article distributed under the terms of the Creative Commons Attribution License (CC BY). The use, distribution or reproduction in other forums is permitted, provided the original author(s) and the copyright owner(s) are credited and that the original publication in this journal is cited, in accordance with accepted academic practice. No use, distribution or reproduction is permitted which does not comply with these terms. 